\title{
Spectral element agglomerate AMGe
}

T. Chartier ${ }^{1}$, R. Falgout ${ }^{2}$, V. E. Henson ${ }^{2}$, J. E. Jones ${ }^{4}$, P. S. Vassilevski ${ }^{2}$, T. A. Manteuffel ${ }^{3}$, S. F. McCormick ${ }^{3}$, and J. W. Ruge ${ }^{3 \star}$

1 Department of Mathematics, Davidson College, P.O. Box 6908, Davidson, NC 28035, U.S.A. email: tichartier@davidson.edu

2 Center for Applied Scientific Computing, UC Lawrence Livermore National Laboratory, P.O. Box 808, L-561, Livermore, CA 94551, U.S.A. email:

$\{$ rfalgout, vhenson, panayot $\}$ @llnl.gov

3 Applied Math Department, Campus Box 526, University of Colorado at Boulder, Boulder, CO 80309-0526, U.S.A. email: \{tmanteuf, stevem, jruge\}@colorado.edu

4 Department of Mathematics, Florida Institute of Technology, 150 West University Blvd., Melbourne, FL, 32901, U. S. A.email: jim@fit.edu

* This work was performed in part under the auspices of the U. S. Department of Energy by University of California Lawrence Livermore National Laboratory under contract W-7405-Eng-48.

\section{Introduction}

In recent years, several extensions of the classical AMG method (see [RS87]) to handle more general finite element matrices have been proposed (see, e.g., [Br00], [JV01], and [Ch03]). Other extensions are related to the so-called smoothed aggregation method, see e.g., [Vn98] and the papers cited therein. For the most recent versions of both the AMG and smoothed aggregation approaches, we refer to [Br05] and [Br04]. In this note, under the assumption that one has access to the fine-grid element matrices, we combine the effectiveness of the element interpolation given in [Br00] with a "spectral" approach to selecting coarse degrees of freedom, as proposed in [Ch03]. The method presented here selects the coarse degrees of freedom from the eigenvectors in the lower parts of the spectra of certain small matricesspecial Schur complements of assembled neighborhood matrices. These Schur complements are associated with so-called minimal intersection sets, which in turn are derived from the partitioning provided by an algorithm (e.g., from [JV01]) that creates agglomerated elements. The idea of selecting coarse degrees of freedom from the eigenvectors of small matrices has been used previously in connection with certain aggregation methods, see, e.g., [FB97] and the preliminary report [Br99].

The remainder of this note consists of the following. In the second section we follow [VZ04] in defining the notion of minimal intersection sets and describe the process of selecting basis vectors which span the coarse vector space. We also prove that the space spanned by the complementary eigenvectors gives rise to a wellconditioned block $A_{f f}$ of the original matrix $A$. The final section contains some numerical illustration of the method. 


\section{The spectral way of selecting coarse degrees of freedom}

Assume we are given the fine-grid symmetric positive (semi-) definite matrix $A$ and have access, for a given set of finite-elements $\{\tau\}$, to the symmetric semi-definite fine-grid element matrices $A_{\tau}$. Here, we consider each element $\tau$ to be a subset (list) of fine-grid degrees of freedom, or dofs. We let $\mathcal{D}$ denote the set of fine dofs, and identify $\mathcal{D}$ with the index set $\{1,2, \ldots, n\}$. For any vector $\mathbf{w}$, we use the notation $\mathbf{w}_{\tau}$ to denote the restriction of $\mathbf{w}$ to the subset $\tau$ of $\mathcal{D}$. Armed with these notational conventions, we note that the original matrix $A$ is assembled from the element matrices in the usual way, that is, for any vector $\mathbf{v}$ one has,

$$
\mathbf{v}^{T} A \mathbf{v}=\sum_{\tau} \mathbf{v}_{\tau}^{T} A_{\tau} \mathbf{v}_{\tau}
$$

Let $\mathbf{V}=V(\mathcal{D})$ denote the vector space (or the space of discrete functions) of vectors with indices from $\mathcal{D}$; that is, one can identify $\mathbf{V}$ with the vector space $\mathbf{R}^{n}$. Based on an agglomeration algorithm (e.g., as originally proposed in [JV01]; see also [Va02]) one generates a set of agglomerated elements $\{T\}$ from the fine-grid elements $\{\tau\}$. Every agglomerated element $T$ consists of a number of connected finegrid elements and every fine-grid element $\tau$ belongs to exactly one agglomerated element $T$.

Note that every agglomerated element $T$ can also be considered as a set of fine degrees of freedom, namely as the union of the fine degrees of freedom that belong to the fine-grid elements $\tau$ that form $T$.

One can partition the fine degrees of freedom (dofs) into non-overlapping sets $\{\mathcal{I}\}$, based on the relationships between agglomerated elements and dofs. This relationship is described by the incidence matrix $\mathcal{E}$, defined as

$$
\mathcal{E}_{i j}=\left\{\begin{array}{l}
1, \text { if dof } j \text { is in the agglomerated element } i, \\
0, \text { otherwise. }
\end{array}\right.
$$

Note that $\mathcal{E} \in \mathbf{R}^{n_{E} \times n}$, where $n_{E}$ is the number of agglomerated elements and $n$ is the number of fine dofs. Consider next $\mathcal{Z}=\mathcal{E}^{T} \mathcal{E} \in \mathbf{R}^{n \times n}$, and observe that $\mathcal{Z}_{i j}=\mid\{T: \quad i \in T$ and $j \in T\} \mid$, where $|\cdot|$ indicates cardinality. That is, $\mathcal{Z}_{i j}$ equals the number of agglomerated elements containing both dofs $i$ and $j$. We then split the set of dofs $\{1, \ldots, n\}$ into non-overlapping sets $\left\{\mathcal{I}_{k}\right\}_{k=1}^{\ell}$ (called minimal intersection sets) with the property that $i$ and $j$ are in one and the same set $\mathcal{I}_{k}$ if and only if $\mathcal{Z}_{i j}=\mathcal{Z}_{i i}=\mathcal{Z}_{j j}$.

With the minimal intersection sets $\mathcal{I}$, one is able to define a change of basis from "nodal" to "spectral" dofs through the following process. For every minimal intersection set $\mathcal{I}$ define the neighborhood $\mathcal{N}(\mathcal{I})=\cup \tau_{\mathcal{I}}$ where the union consists of all fine elements $\tau_{\mathcal{I}}$ that share a dof from $\mathcal{I}$. Let the assembled local matrix be denoted $A_{\mathcal{N}(\mathcal{I})}$ and compute its Schur complement $S_{\mathcal{I}}$ by eliminating the dofs outside $\mathcal{I}$. (Note that in the case where $\mathcal{I}$ is a single dof and $A_{\mathcal{N}(\mathcal{I})}$ is a semidefinite matrix this Schur complement may be the zero matrix). Next, we compute all the eigenvalues of $S_{\mathcal{I}}$ and the associated eigenvectors $\left\{\mathbf{q}_{\mathcal{I} ; k}\right\}, k=1, \ldots,|\mathcal{I}|$. Whenever necessary we extend the eigenvectors by zero outside $\mathcal{I}$. If $S_{\mathcal{I}}=[0]$ we use the standard unit vectors $\mathbf{q}_{\mathcal{I} ; k}:=\mathbf{e}_{i}$ for $i \in \mathcal{I}$. We may observe that the set $\left\{\mathbf{q}_{\mathcal{I} ; k}\right\}, k=1, \ldots,|\mathcal{I}|$, for $\mathcal{I}$ running over all the minimal intersection sets, provides an orthogonal basis of $\mathbf{R}^{n}$. For a given minimal intersection set $\mathcal{I}$ the group 
of vectors $\left\{\mathbf{q}_{\mathcal{I} ; k}\right\}$ are orthogonal (as eigenvectors of symmetric matrices), and if two vectors belong to groups for different sets $\mathcal{I}$ they have non-intersecting supports; therefore they are also orthogonal.

For every set $\mathcal{I}$ we split the eigenvectors into two groups $\mathbf{V}_{\mathcal{I}_{c}}$ and $\mathbf{V}_{\mathcal{I}_{f}}$ in the following way. Let the eigenvectors $\left\{\mathbf{q}_{\mathcal{I} ; k}\right\}$ be ordered according to the eigenvalues $0 \leq \lambda_{1} \leq \lambda_{2} \leq \ldots \leq \lambda_{|\mathcal{I}|}$. For any given integer $1 \leq p_{\mathcal{I}} \leq|\mathcal{I}|$, the first $p_{\mathcal{I}}$ eigenvectors define the orthogonal basis of the space $\mathbf{V}_{\mathcal{I}_{c}}$. This space, representing the set of " $c$ "-dofs (or coarse dofs), corresponds to a lower portion of the spectrum of the Schur complement $S_{\mathcal{I}}$. The remaining eigenvectors span $\mathbf{V}_{\mathcal{I}_{f}}$. Together, they form an orthogonal decomposition of the space spanned by the eigenvectors for $S_{\mathcal{I}}$, and taking all such spaces for all $\mathcal{I}$ we observe that

$$
\mathbf{R}^{n}=\mathbf{V}=\mathbf{V}_{\mathcal{I}_{c}} \bigoplus_{\mathcal{I}} \mathbf{V}_{\mathcal{I}_{f}}
$$

We denote the direct sums, over all $\mathcal{I}$, of the coarse and fine spaces, respectively, as $\mathbf{V}_{c}$ and $\mathbf{V}_{f}$. The motivation for this splitting is given next.

Lemma 1. The block $A_{f f}$, representing the restriction of $A$ to the subspace $\mathbf{V}_{f}$, is well-conditioned if $p_{\mathcal{I}}$ for all $\mathcal{I}$ are sufficiently large, in particular if $\lambda_{p_{\mathcal{I}}+1}\left[S_{\mathcal{I}}\right]>0$ and $\lambda_{p_{\mathcal{I}}+1}\left[S_{\mathcal{I}}\right] \simeq\left\|A_{T}\right\|$ for all neighboring $T$ that contain $\mathcal{I}$.

Proof. Define the vector norm $\|$.$\| as \|\mathbf{w}\|=\sqrt{\mathbf{w}^{T} \mathbf{w}}$. Let $\mathbf{v}_{f} \in \mathbf{V}_{f}$, that is, $\mathbf{v}=\mathbf{v}_{f}$ is a vector with vanishing coarse-grid component. We can split the inner product $\mathbf{v}^{T} A \mathbf{v}$ over the agglomerates $T$, using the local matrices $A_{T}$, and then rewrite the $\operatorname{sum} \sum_{T} \mathbf{v}_{T}^{T} A_{T} \mathbf{v}_{T}=\sum_{\mathcal{I}} \sum_{T: \mathcal{I} \subset T} C_{\mathcal{I}} \mathbf{v}_{T}^{T} A_{T} \mathbf{v}_{T}$ for some constants $C_{\mathcal{I}}$ (depending on the number of agglomerates $T$ that share $\mathcal{I}$ ). Using well known minimization properties of Schur complements of symmetric positive (semi-)definite matrices, one readily obtains the inequalities

$$
\min _{\mathcal{I}} C_{\mathcal{I}} \lambda_{p_{\mathcal{I}}+1}\left[S_{\mathcal{I}}\right]\left\|\mathbf{v}_{f}\right\|^{2} \leq \mathbf{v}_{f}^{T} A_{f f} \mathbf{v}_{f} \leq \max _{T}\left\|A_{T}\right\|\left\|\mathbf{v}_{f}\right\|^{2} . \text { QED }
$$

Remark 1. The $C_{\mathcal{I}}$ are topological constants (i.e., independent of the matrix). However, we have the option to choose the integers $p_{\mathcal{I}}$ sufficiently large to lead to an improved minimal eigenvalue of $A_{f f}$. Hence, by selecting the $p_{\mathcal{I}}$ appropriately, we can insure that $A_{f f}$ is well conditioned. We may also observe that for the model case where $A$ is the discretization of the 2D finite element Laplacian, both bounds in (1) are mesh independent. The property that $A_{f f}$ is well conditioned gives rise to a special form of the so-called compatible relaxation principle introduced in [B00].

In the new (orthogonal) basis, the matrix $A$ has a block, $A_{f f}$, that is wellconditioned. With the coarse dofs identified, the interpolation matrix $P$ can be computed locally by building, for every agglomerated element $T$, a prolongator $P_{T}$ such that fine dofs that are shared by two or more agglomerated elements are interpolated by the coarse dofs from that common set. More specifically, for every dof $i$ consider its neighborhood $N(i)=\cap\{T: i \in T\}$. Then $i$ is interpolated from all coarse dofs that belong to $N(i)$. Further details about this construction are found in [JV01].

It can be proven, in the manner given in [JV01], that the locally constructed $P$ satisfies a weak approximation property. That is, for some constant $\eta \geq 1$, 


$$
\|A\|\left\|\mathbf{v}-P \mathbf{v}_{c}\right\|^{2} \leq \eta \mathbf{v}^{T} A \mathbf{v} \text { for any } \mathbf{v}=\mathbf{v}_{f}+\mathbf{v}_{c},
$$

where $\mathbf{v}_{f} \in \mathbf{V}_{f}, \mathbf{v}_{c} \in \mathbf{V}_{c}$. This implies (see e.g., [Br00]) an optimal convergence result for a two-grid method based on $P$ and simple Richardson smoothing.

\section{Numerical Experiments}

We describe here results of numerical experiments designed to illustrate the use of the spectral agglomerate AMGe algorithm. We first stress that, in 2D, the minimal intersection sets are the vertices of the agglomerated elements (all of which naturally become coarse dofs), the interior of the faces of the agglomerated elements (or AEfaces), as well as the interior of the agglomerated elements (or AEs). For either of these two minimal intersection sets, we can form the appropriate Schur complement, $S_{A E f}$ or $S_{A E}$, and compute the associated eigenvalues and eigenvectors.

For the sets of eigenvectors of $S_{A E f}$ or $S_{A E}$, we select an eigenvector with index $k$ to define a coarse dof if, given a tolerance $\tau \in[0,1)$, the corresponding eigenvalue $\lambda_{k}$ satisfies

$$
\lambda_{k}<\tau \lambda_{\max }
$$

We have the option to use different values of $\tau$ for the sets of eigenvectors; one, denoted $\tau_{A E f}$, is used on the eigenvectors of $S_{A E f}$, while another one (denoted by $\left.\tau_{A E}\right)$ is applied to select coarse dofs from the eigenvectors of $S_{A E}$.

To save some computation in the setup phase one may choose to set $\tau_{A E}=0$. (Note that when $\tau=0$ for both sets the resulting spectral agglomerate AMGe reduces to the agglomeration based AMGe method from [JV01]; that is, the coarse dofs are the vertices of the agglomerates only.) In general, we recommend choosing $\tau_{A E}<\tau_{A E f}$.

In $3 \mathrm{D}$ there are additional minimal intersection sets: subsets of the boundary of the faces of the agglomerated elements. We also select these sets as coarse dofs. For thin body elasticity (the particular 3D application we consider in this section) choosing these additional sets as coarse dofs is acceptable in terms of computational complexity.

The problems utilized for this study are:

(1) a $\mathbf{2 D}$ anisotropic diffusion problem, given by

$$
-\operatorname{div}\left(\left(\epsilon I+\underline{b b}^{T}\right) \nabla u\right)=f,
$$

posed in a unit square. Three different selections for parameter $\epsilon$ are considered, $\epsilon=1,0.01,0.001$, which control the strengths of the anisotropy in each experiment. The anisotropy is not grid aligned, and its direction is controlled by $\underline{b}=\left[\begin{array}{c}\cos \theta \\ \sin \theta\end{array}\right], \theta=\frac{\pi}{4}$. For this $2 \mathrm{D}$ problem, results computed on unstructured triangular meshes with 6400 and 25600 elements are presented. A mesh typical of the problem, with 1600 elements, is shown in Fig. 1 (left).

(2) a $3 \mathrm{D}$ thin body elasticity problem posed on a domain $\Omega=(0,1) \times(0,1) \times$ $(0, d)$ and discretized on a number of uniform trilinear hexahedral meshes. The problem is formulated as follows: for a given vector function $\mathbf{f}=\left(f_{i}\right)_{i=1}^{3}$, find the displacements $\mathbf{u}=\left(u_{i}\right)_{i=1}^{3}$ such that, for all $\mathbf{x}=\left(x_{i}\right)_{i=1}^{3} \in \Omega$ 


$$
\sum_{j=1}^{3} \frac{\partial}{\partial x_{j}}\left(\sum_{k, l=1}^{3} E_{i, j, k, l} \frac{\partial u_{k}(\mathbf{x})}{\partial x_{l}}\right)=f_{i}(\mathbf{x})
$$

for $i=1,2,3$. Homogeneous boundary conditions are imposed: A Dirichlet condition of $u_{i}=0, i=1,2,3$ is imposed on the side of the body $\Gamma_{D}=\{(x, y, z=0)\}$, and, on the remainder of the boundary $\partial \Omega \backslash \Gamma_{D}$, we apply a Neumann condition

$$
\sum_{j=1}^{3} n_{j}\left(\sum_{k, l=1}^{3} E_{i, j, k, l} \frac{\partial u_{k}(\mathbf{x})}{\partial x_{l}}\right)=0
$$

for $i=1,2,3$. Here, $\mathbf{n}=\left(n_{i}\right)_{i=1}^{3}$ is the outward unit normal to $\partial \Omega$. The coefficients $E_{i, j, k, l}$ are expressed in terms of the Lame coefficients $\lambda=113$ and $\mu=81$ as follows:

$$
\begin{aligned}
E_{1,1,1,1}=E_{2,2,2,2}=E_{3,3,3,3} & =2 \mu+\lambda \\
E_{i, i, j, j} & =\lambda, \quad i \neq j, i, j=1,2,3 \\
E_{i, j, i, j}=E_{j, i, i, j}=E_{i, j, j, i}=E_{j, i, j, i} & =\mu, \quad i \neq j, i, j=1,2,3 \\
E_{i, j, k, l} & =0, \quad \text { all remaining indices. }
\end{aligned}
$$

We describe results for this problem using several different geometries for the body. Using the basic mesh size $h=0.25$, we observe results for four different choices of $d$, namely $d=25 h=6.25, d=50 h=12.5, d=100 h=25$ and $d=200 h=50$. The geometry for this problem is shown in Fig. 1 (right).
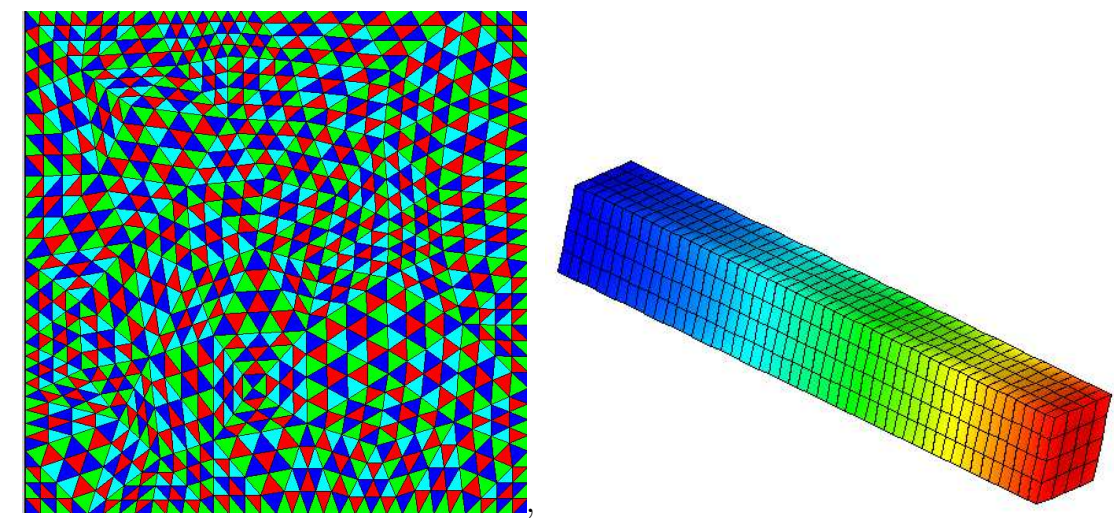

Fig. 1.

Unstructured triangular mesh: 1600 elements (left) Tri-linear hexahedral mesh for 3D elasticity problem (right).

For all experiments we apply one iteration of symmetric block Gauss-Seidel as the smoother in a $\mathrm{V}(1,1)$-cycle. The blocks in the smoother correspond to the elements of the grid at given level and hence are overlapping. 
The results of the experiments are given in the several tables that follow. For each experiment, we report several quantities: the number of iterations needed to reduce the $\ell^{2}-$ norm of the initial residual by $10^{-9}$; the asymptotic reduction factor $\rho$; the grid and operator complexities; and, for the 3D case, the number of coarsening levels used in the problem. The grid and operator complexities, commonly used in AMG, are defined respectively as the total number of dofs on all levels divided by the number of dofs on the finest level and the total number of nonzero entries in the operator matrices for all levels divided by the number of nonzero entries in the fine level matrix $A$. The tables also indicate the mesh sizes and values for parameters of the algorithm (such as $\tau$ ).

Table 1. Convergence results for AMGe and the spectral agglomerate AMGe with $\tau_{A E}=$ $\frac{1}{4} \tau_{A E f}=\frac{1}{4} \tau ; 2 \mathrm{D}$ anisotropic diffusion.

\begin{tabular}{|c|c|c|c|c|c|}
\hline $\begin{array}{l}6400 \text { elements } \\
10 \text { levels }\end{array}$ & $\begin{array}{l}\text { AMGe } \\
(\tau=0)\end{array}$ & $\begin{array}{r}\text { Spec } \\
\tau=0.0312\end{array}$ & $\begin{array}{l}\text { agglor } \\
=0.12\end{array}$ & $\begin{array}{l}\text { ate } \mathrm{AN} \\
=0.2\end{array}$ & $\begin{array}{l}\mathrm{e} \\
\tau=0.5\end{array}$ \\
\hline iterations & 12 & 10 & 9 & 8 & 6 \\
\hline & 0.405 & 0.432 & 0.396 & 0.313 & 0.176 \\
\hline grid complexity & 1.64 & 2.77 & 2.79 & 2.86 & 3.13 \\
\hline operator complexity & 1.86 & 4.73 & 4.81 & 5.13 & 6.83 \\
\hline iterations & 25 & 16 & 11 & 9 & 7 \\
\hline & 0.614 & 0.511 & 0.378 & 0.306 & 0.185 \\
\hline grid complexity & 1.64 & 2.86 & 3.11 & 3.34 & 3.76 \\
\hline operator complexity & 1.86 & 5.09 & 6.31 & 7.99 & 14.01 \\
\hline$\epsilon=0.001 \quad$ iterations & 36 & 17 & 15 & 13 & 13 \\
\hline & 0.721 & 0.517 & 0.428 & 0.388 & 0.365 \\
\hline grid complexity & 1.64 & 3.09 & 3.31 & 3.50 & 3.87 \\
\hline operator complexity & 1.86 & 6.11 & 7.46 & 9.32 & 16.18 \\
\hline
\end{tabular}

The numerical results generally agree with the observations in Remark 1; it is clear that richer coarse spaces produce better the convergence factors. Naturally, this gain is obtained at the expense of higher complexities of the method. The spectral agglomerate AMGe method can become a fairly expensive method; it requires a number of local computations: assembling of local neighborhood matrices, computing their respective Schur complements $S_{A E}$ and $S_{A E f}$, and solving local eigenproblems associated with them. In addition to this, all the normal costs of the traditional AMG-type methods applies; namely, computing the respective interpolation matrices and the associated coarse-level stiffness matrices. The local dense matrices grow in size with the tolerance $\tau$. This cost is especially noticeable in 3D problems, where the increased complexity leads to significant increases in the time required to solve the problem. steps (e.g., between 5 and 20) The operator complexities can be reduced by using more aggressive agglomeration at the initial level(s). 
Table 2. Convergence results for AMGe and the spectral agglomerate AMGe with $\tau_{A E}=$ $\frac{1}{4} \tau_{A E f}=\frac{1}{4} \tau ; 2 \mathrm{D}$ anisotropic diffusion.

\begin{tabular}{|c|c|c|c|c|c|}
\hline $\begin{array}{c}25600 \text { elements } \\
12 \text { levels }\end{array}$ & $\begin{array}{l}\text { AMGe } \\
(\tau=0)\end{array}$ & \multicolumn{4}{|c|}{ Spectral agglomerate AMGe } \\
\hline iterations & 15 & 22 & 21 & 10 & 6 \\
\hline & 0.517 & 0.673 & 0.649 & 0.429 & 0.154 \\
\hline grid complexity & 1.62 & 2.81 & 2.82 & 2.92 & 3.21 \\
\hline operator complexity & 1.86 & 5.01 & 5.04 & 5.62 & 8.41 \\
\hline iterations & 32 & 30 & 23 & 12 & 8 \\
\hline & 0.715 & 0.747 & 0.673 & 0.431 & 0.202 \\
\hline grid complexity & 1.62 & 2.90 & 3.01 & 3.42 & 3.89 \\
\hline operator complexity & 1.86 & 5.43 & 5.96 & 9.60 & 21.92 \\
\hline$\epsilon=0.001 \quad$ iterations & 57 & 36 & 28 & 22 & 20 \\
\hline & 0.834 & 0.761 & 0.698 & 0.604 & 0.549 \\
\hline grid complexity & 1.62 & 3.13 & 3.25 & 3.62 & 4.04 \\
\hline operator complexity & 1.86 & 6.58 & 7.36 & 12.25 & 28.38 \\
\hline
\end{tabular}

Table 3. Convergence results for spectral agglomerate AMGe with $\tau_{A E}=\frac{1}{4} \tau_{A E f} ; 3 \mathrm{D}$ thin body elasticity.

\begin{tabular}{|r||ccccc|}
\hline \multicolumn{1}{|c||}{$\begin{array}{c}\text { S00 elements } \\
d=25 h\end{array}$} & \multicolumn{5}{c|}{ Spectral agglomerate AMGe } \\
\hline \hline iterations & 29 & 28 & 28 & 15 & 7 \\
$\rho$ & 0.748 & 0.738 & 0.727 & 0.553 & 0.295 \\
grid complexity & 2.20 & 2.22 & 2.28 & 2.50 & 3.28 \\
operator complexity & 2.84 & 2.90 & 3.04 & 3.63 & 6.94 \\
coarsening levels & 7 & 7 & 7 & 7 & 7 \\
\hline
\end{tabular}

Table 4. Convergence results for spectral agglomerate AMGe with $\tau_{A E}=\frac{1}{4} \tau_{A E f} ; 3 \mathrm{D}$ thin body elasticity.

\begin{tabular}{|c||ccccc|}
\hline \multicolumn{1}{|c||}{1600 elements } & \multicolumn{5}{c|}{ Spectral agglomerate AMGe } \\
$d=100 h$ & $\tau=0.03125$ & $\tau=0.0625$ & $\tau=0.125$ & $\tau=0.25$ & $\tau=0.5$ \\
\hline \hline iterations & 39 & 32 & 28 & 15 & 7 \\
$\rho$ & 0.860 & 0.788 & 0.739 & 0.555 & 0.264 \\
grid complexity & 2.09 & 2.12 & 2.18 & 2.41 & 3.25 \\
operator complexity & 2.53 & 2.60 & 2.73 & 3.36 & 6.81 \\
coarsening levels & 9 & 9 & 9 & 9 & 9 \\
\hline
\end{tabular}




\section{Conclusions}

The purpose of this note is to describe an algorithm resulting from the uniting of two ideas introduced and applied elsewhere. For many problems, AMG has always been difficult due to complexities whose natures are difficult to discern from the entries of matrix $A$ alone. Element-based interpolation has been shown to be an effective method for some of these problems, but it requires access to the element matrices on all levels. One way to obtain these has been to perform element agglomeration to form coarse elements, but in complicated situations defining the coarse dofs in not easy. The spectral approach to coarse dof selection is very attractive due to its elegance and simplicity. The algorithm presented here combines the robustness of element interpolation, the ease of coarsening by element agglomeration, and the simplicity of defining coarse dofs through the spectral approach. As demonstrated in the numerical results, the method does yield a reasonable solver for the problems described. It can, however, be an expensive method due to the number and cost of the local, small dense linear algebra problems; making it a generally competitive method remains an area for further research.

\section{References}

[B00] Brandt, A.: Generally highly accurate algebraic coarsening, Elec. Trans. Num. Anal., 10, 1-20 (2000)

[Br00] Brezina, M, Cleary, A. J., Falgout, R. D., Henson, V. E., Jones, J. E., Manteuffel, T. A., McCormick, S. F., and Ruge, J. W.: Algebraic multigrid based on element interpolation (AMGe). SIAM Journal on Scientific Computing, 22, 1570-1592 (2000)

[Br99] Brezina, M, Heberton, C., Mandel, J., and Vaněk, P.: An iterative method with convergence rate chosen a priori. UCD/CCM Report 140, (1999)

[Br04] Brezina, M., Falgout, R., MacLachlan, S., Manteuffel, T., McCormick, S., and Ruge, J.: Adaptive smoothed aggregation (aSA). SIAM J. Sci. Comp., 25, 1896-1924 (2004)

[Br05] Brezina, M., Falgout, R., MacLachlan, S., Manteuffel, T., McCormick, S., and Ruge, J.: Adaptive algebraic multigrid methods. SIAM J. Sci. Comp., submitted (2004)

[Ch03] Chartier, T., Falgout, R. D., Henson, V. E., Jones, J. E., Manteuffel, T. A., Mc-

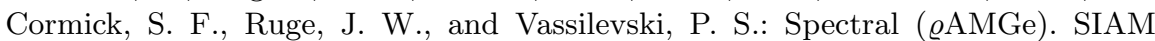
Journal on Scientific Computing, 25, 1-26 (2003)

[FB97] Fish, J. and Belsky, V.: Generalized aggregation multilevel solver. Intern. J. Numer. Methods in Engineering, 40, 4341-4361(1997)

[JV01] Jones, J. E. and Vassilevski, P. S.: AMGe based on element agglomeration. SIAM Journal on Scientific Computing, 23, 109-133(2001)

[RS87] Ruge, J. W. and Stüben, K.: Algebraic multigrid (AMG). In: McCormick, S. F. (ed) Multigrid Methods, vol. 3 of Frontiers in Applied Mathematics, SIAM, Philadelphia, PA, 73-130 (1987)

[Vn98] Vaněk, P., Mandel, J., and Brezina, M.: Convergence of algebraic multigrid based on smoothed aggregation. Numerische Mathematik, 88, 559-579(2001)

[Va02] Vassilevski, P. S.: Sparse matrix element topology with application to AMG and preconditioning. Numer. Linear Algebra Appl., 9, 429-444(2002)

[VZ04] Vassilevski, P. S. and Zikatanov, L. T.: Multiple vector preserving interpolation mappings in algebraic multigrid. Lawrence Livermore National Laboratory Technical Report UCRL-JRNL-208036, November (2004) 